\title{
Körper, Tod und Flüssigkeiten - Das Abjekte der Organspende
}

\author{
Annerose Böhrer
}

Eingegangen: 30. Mai 2021 / Angenommen: 27. August 2021 / Online publiziert: 1. Oktober 2021

(C) Der/die Autor(en) 2021

Zusammenfassung Die postmortale Transplantation von Organen gilt als integraler Bestandteil von Hochleistungsmedizin. Zugleich wird immer wieder ein Mangel an gespendeten Organen problematisiert und die Autonomie der Patientinnen und Patienten gegen einen gesellschaftlichen Solidaritätsanspruch gestellt. Eine zentrale Praxis in diesem Zusammenhang ist die ,Aufklärung' der Bevölkerung. In den für diesen Zwecken zusammengestellten Informationen und Kampagnen finden Widerspruch und Unbehagen jedoch meistens keinen Raum und auch bestimmte Aspekte der Organspende (wie z. B. der Tod der Spender/-innen) werden tendenziell oft übergangen. Der Beitrag richtet das Augenmerk auf eben jene ambivalenten Bereiche der Organspende und schlägt vor, sich diesen mit Kristevas Konzept des Abjekten zu nähern. Das ,Abjekte der Organspende“ ließe sich demnach auf vier Ebenen verorten, die vor allem die Körper der Spender/-innen betreffen: Tod und Sterben, die Zergliederung des Körpers, die Organe und das Körperinnere sowie die in der Praxis der Organspende entstehenden Grenzverschiebungen bzw. -auflösungen. Das Wechselspiel der Annäherung an und Distanzierung von diesen Aspekten der Körperlichkeit und des Sterbens, das in den Kulturtechniken der Organspende sichtbar wird, ist aus dieser Perspektive ein integraler Bestandteil dieser Praxis im Umgang mit Körper, Tod und Sterben.

Schlüsselwörter Transplantation $\cdot$ Abjekt $\cdot$ Körpersoziologie $\cdot$ Sterben $\cdot$ Metaphern

Annerose Böhrer $(\square)$

Friedrich-Alexander-Universität Erlangen-Nürnberg, Kochstraße 4, 91052 Erlangen, Deutschland

E-Mail: anne.boehrer@fau.de 


\section{Body, death and fluids-Abjection in organ donation}

Abstract The post-mortem transplantation of organs is considered an integral component of high-performance medicine. At the same time, a shortage of donated organs is repeatedly problematized and the autonomy of patients is set against a social claim to solidarity. A central practice in this context is to inform and educate the population. In the campaigns and information compiled for this purpose, however, there is usually no room for contradiction and discomfort and certain aspects of organ donation (such as the death of donors) also tend to be widely ignored. This article focuses on those ambivalent areas of organ donation and proposes to approach them with Kristeva's concept of abjection. The "abjection in organ donation" could thus be located on four levels, which primarily affect the bodies of the donors: death and dying, the dissection of the body, the organs and the inside of the body as well as the shifting or dissolution of boundaries that occur in the practice of organ donation. From this perspective, the interplay of approaching and distancing from these aspects of corporeality and of dying, which is visible in the cultural techniques of organ donation, is an integral component of this practice in dealing with body, death and dying.

Keywords Transplantation · Abjection - Sociology of the body · Dying · Metaphors

\section{Einleitung}

Die postmortale Transplantation von Organen gilt - obgleich sie relativ selten durchgeführt wird - als integraler Bestandteil medizinisch-therapeutischer Bemühungen um Menschen mit terminalem Organversagen. ${ }^{1}$ Immer wieder wird in diesem Kontext ein Mangel an gespendeten Organen problematisiert. Im empirischen Material zur Organspende-Kommunikation durch entsprechende Organisationen (z. B. Bundezentrale für gesundheitliche Aufklärung (BZgA), Deutsche Stiftung Organtransplantation (DSO) oder Patient:innenverbände, scheint oft die Idee auf, eine ,rationale' und zugleich stark informationsreduzierte Aufklärung müsse überzeugen und zu einer höheren Spendenbereitschaft führen. Widerspruch und Unbehagen finden hier wenig Raum und werden allenfalls als zu lösendes Problem adressiert. Bestimmte Aspekte der Organspende (etwa der Tod und die Öffnung des Körpers) eignen sich offenkundig nicht für eine werbewirksame Kommunikation und werden aus Kampagnen und Veranstaltungen wie dem ,Tag der Organspende " weitgehend ausgeschlossen.

Eben jener (vermeintliche) Ausschluss soll uns an dieser Stelle interessieren und kritisch befragt werden. Mit Bernhard Giesen (2010), der betont, eine Grenze konsti-

\footnotetext{
1 Aus den Statistiken der DSO geht hervor, dass jährlich nur etwa 1000 Menschen als potenzielle Organspender:innen identifiziert werden, was unter anderem damit zusammenhängt, dass nur hirntote Patient:innen für eine Spende in Frage kommen. Im Jahr 2020 waren es laut DSO 1034 potenzielle Spender:innen (DSO 2020).
} 
tuiere sich immer auch durch das, was außerhalb der Begrenzung liege (S. 39), lässt sich bereits erahnen, dass das, was wir in Formaten öffentlicher Gesundheitskommunikation nicht finden, keineswegs ,nicht vorhanden“ ist. Julia Kristevas (1982) theoretische Konzeption des Abjekten soll dabei den Blick für das kulturkonstituierende Moment der Abjektion öffnen. Die Dynamik des Ausschlusses bzw. der Abjektion ist aus dieser Perspektive keine destruktive Kraft, sondern erschafft vielmehr komplexe materielle und immaterielle Gefüge, die wir als Kulturen der Organspende untersuchen können.

Für diesen Artikel greife ich auf Materialien öffentlicher Gesundheitskommunikation als Teil des Datenkorpus meiner Dissertation (Erhebung 2016-20) zurück, die Organspende als gesellschaftliche Praxis mit einem besonderen Augenmerk auf Materialität (Mol 2002; Woodward 2019) und Metaphern (Lakoff und Johnson 2003) erforscht.

\section{Das Abjekte der Organspende}

Zentraler Bestandteil der Organspende als gesellschaftliche Praxis ist die ,Aufklärung ' der Bevölkerung (Bundesministerium der Justiz und für Verbraucherschutz/ Bundesamt für Justiz 2007, 1/§ 2). Darunter subsummieren sich immer wieder neue Auflagen von Plakat- und anderen Medienkampagnen (Schaper et al. 2018; Hansen und Schicktanz 2018; Hansen et al. 2020), die Produktion von Informationsmaterialien und das Durchführen von Informationsveranstaltungen. Gerade im Feld gesundheitlicher Aufklärung erscheint die postmortale Transplantation meist weder als besonders ,blutige Angelegenheit' noch als Ereignis, das mit dem Tod der potenziellen Spender/-innen in Verbindung stünde. In Deutschland scheint die Frage nach Organspenden derzeit vor allem ein bürokratisches Anliegen zu sein, das mit dem Besitz des Organspendeausweises einhergehen soll: Die aktuelle Plakatkampagne der BZgA bildet weder Körper noch Organe oder Menschen ab, sie spricht nicht über den Tod, sondern zeigt Organspendeausweise, gefaltet in Form von Alltagsgegenständen, wie Handtaschen, Kopfhörern oder Kleidern (BZgA 2016).

Andere Kampagnen konzentrieren sich auf den Aspekt der Spende und rahmen die Vorgänge der postmortalen Transplantation als ,Gabe‘. ${ }^{2}$ Diese Rahmung findet sich nicht nur im deutschen Sprachraum (vgl. engl.: ,donation'). Während wir in den deutschen Kampagnen eine Kombination aus Ausweis und Alltagsgegenständen finden, ließ sich die Organisation Donate Life America im Jahr 2019 für ihre Organspende-Kampagnen von dem Satz ,life is a beautyful ride“ als Motto für den sogenannten ,National Donate Life Month“ inspirieren. Das Fahrrad auf den Bildern stünde wie die ,transplanting journey“ als Symbol für Erneuerung und den ,circle of life“" (New Hannover Regional Medical Center o.J.). Hatten wir es hier zunächst mit einem eher ,technischen' Alltagsgegenstand zu tun, so wandten sich die Kampagnen der gleichen Einrichtung im Jahr 2020 einem neuen Thema zu: Der Natur als

\footnotetext{
2 So beispielsweise die Pro-Kampagne des Deutschen Herzzentrums von 2010. Die Motive zeigten die Gesichter von Prominenten, die ihren Blick direkt in die Kamera richteten, darunter der Slogan: „Du bekommst alles von mir, ich auch von Dir?“ (Danek 2010; Arp 2011).
} 
„Garden of Life“. Inspiriert von einer frühlingshaften Gartenszene sollen Garten und Insekten für „hope, courage and transformed life“ stehen (Donate Life o.J.). Tod, Körper und die Notwendigkeit ihrer Zergliederung tauchen in dieser Art der Kommunikation nicht auf. Auch in der deutschen Plakatkampagne erscheint der ,Tod (der auf dem Ausweis immerhin zweimal erwähnt wird) nicht, denn in den meisten Plakatmotiven wird das Wort durch die Faltung verdeckt. Was wir stattdessen sehen, sind Worte wie „Organspendeausweis“, „Transplantationsgesetz“, „Ja, ich erlaube“ oder vereinzelt das Wort „Nein“ (BZgA o.J.). Die Kampagne hebt, so könnte man sagen, bestimmte Aspekte des deutschen Transplantationssystems hervor: die aktive Zustimmung, Versuche der Normalisierung, eine individuelle Entscheidung und ein dafür geschaffenes Dokument.

Die Erkenntnis über die Vermeidung bestimmter Themenkomplexe, die als abschreckend oder unangenehm gelten, ist keineswegs neu. Lesley Sharp (2007) macht in ihrer langjährigen Forschung zur Organspende in den Vereinigten Staaten ähnliche Beobachtungen und kritisiert, der Tod sei vor allem durch seine auffällige Abwesenheit präsent: „Even more troubling is the fact that the ideology driving organ transfer in this country regularly celebrates life while denying death's presence“ (Sharp 2007, S. 24). Auch die Namen der mit Transplantation befassten Organisationen (Gift of Life, LifeCenter, LifeBanc, Life Connection, Lifeline, Life Choice etc., vgl. Sharp 2007, S. 14) würden, so kritisiert sie, die Anwesenheit des Todes leugnen. Ähnliches konstatiert Anja Jensen (2011) über die dänische Informationspolitik, wenn sie schreibt:

Public campaigns and information about organ donation produced by the Danish Health Agency tend to focus more on public attitudes regarding "yes" or „no“" than thoughts about the procedure itself. This might indicate that policy makers themselves regard the information so precarious that it is dangerous to inform too much about. (Jensen 2011, S. 171).

Auch andere Autorinnen und Autoren kritisieren die Kommunikationspolitik(en) der Organspende (u.a. Kalitzkus 2009; Amelang 2014; Bea 2017; Hansen und Schicktanz 2018). Anschließend an diesen kritischen sozialwissenschaftlichen Diskurs möchte ich vorschlagen, die Praktiken des Feldes mit Kristevas Konzept des Abjekten bzw. der Abjektion zu betrachten und damit den Blick nicht nur für das defizitäre Moment einer mangelhaften Kommunikation, sondern auch für das kulturkonstituierende Moment zu öffnen.

Was aber ist Abjektion? Und wie ließe sich das Konzept auf die postmortale Transplantation anwenden? Kristeva (1982) entwickelt in ,The Powers of Horror ${ }^{6}$ eine psychoanalytisch argumentierende Kulturtheorie, die sich dem Abjekten und den Prozessen seiner systematischen Ausgrenzung widmet und damit allem, was das Subjekt selbst und unsere symbolischen Ordnungen bedroht. Im Mittelpunkt ihres Interesses steht der Zusammenhang von Sprache, Bedeutung und Körper: Bedeutung wird als dem Körper inhärent angenommen (Kristeva 1982, S. 10). Das Abjekte ist dabei weder Subjekt noch Objekt, sondern beschreibt vielmehr eine Beziehung zu jenem Ausgeschlossenen, Auszuschließenden, die wir eingehen müssen, um uns zu schützen (Kristeva 1982, S. 135). Sie beschreibt dies als: 
Not me. Not that. But not nothing, either. A ,something“ that I do not recognize as a thing. A weight of meaninglessness, about which there is nothing insignificant, and which crushes me. On the edge of nonexistence and hallucination, of a reality that, if I acknowledge it, annihilates me. (Kristeva 1982, S. 2).

Kategorien und binäre Unterscheidungen (wie ,tot' oder ,lebendig '), die normalerweise unser kulturelles Leben organisieren und Erfahrungen Bedeutung verleihen, können in Konfrontation mit dem Abjekten durcheinandergeraten und die eigene Fragilität bewusst machen. Diese Bedrohung kann als beängstigend und aufregend zugleich wahrgenommen werden. Die Prozesse der Abjektion dienen dabei als eine Art kulturelle Sicherheitsmaßnahme: ,abject and abjection are my safeguards. The primers of my culture“ (Kristeva 1982, S. 2).

Der Kontakt mit dem Abjekten wird jedoch nicht einfach unterbunden, sondern immer wieder rituell erneuert (Kristeva 1982, S. 4f.). Ähnlich wie mit dem Tod verhält es sich mit Fäkalien und vielen unserer Körperflüssigkeiten, die fortwährend produziert werden, sie sind Bestandteile unserer Körper und damit von uns selbst. Dennoch sind sie meist nicht Teil unserer Alltagsgespräche. Zugleich gelingt ihr Ausschluss nie vollständig. Vielmehr sind sie eingebunden in ein (im)materielles Netzwerk kultureller Techniken: Wassersysteme, Lufterfrischer, räumliche Begrenzungen oder Umschreibungen wie ,wir würden uns noch schnell die Hände waschen". Innerhalb eines bestimmten kulturellen Rahmens ist das Abjekte also durchaus zulässig, mitunter sogar explizit akzentuierbar. So erscheinen in Horrorfilmen Blut, Gewalt, Schmerz und innere Organe zu Zwecken der Unterhaltung gut aufgehoben; das (potenziell) Abjekte ist in dieser kulturellen Bearbeitung ,gezähmt " und eingehegt in das Genre eines Unterhaltungsformats.

Was abjekt ist oder wo Prozesse der Abjektion stattfinden, lässt sich - und hier liegt eine Schwierigkeit dieses Ansatzes für die empirische Forschung - vor allem da erkennen, wo es (fast) abwesend ist. Für das Phänomen der Organspende bedeutet dies vor allem, den Blick auf die Diskrepanz zwischen der Praxis der Organtransplantation, wie sie im medizinisch-professionellen Bereich stattfindet, und der Organspende als öffentliche Praxis zu richten, die sich in Deutschland vorrangig durch das Bewerben und Besitzen von Organspendeausweisen vollzieht. So wird sichtbar, wie bestimmte Aspekte, die für die Organspende grundlegend sind (z. B. die Mortalität und Materialität der Körper) oft nur angedeutet oder in Bildern und Metaphern umschifft werden: Beispielsweise wenn Organspende durch das Bild eines Ausweises dargestellt und das Wort ,Tod' (wie oben erwähnt) in den Posterkampagnen aus dem Bild gefaltet wird.

Das Abjekte der Organspende lässt sich, so möchte ich vorschlagen, auf vier Ebenen verorten, die vor allem die Körper der Spender:innen betreffen und sich durch ihre (fast) Abwesenheit in öffentlich-affirmativen Praktiken der Organspende auszeichnen:

1. Ist die Ebene von Tod und Sterben für die postmortale Transplantation zugleich Voraussetzung und Hindernis. Voraussetzung, weil das Transplantationsgesetz (TPG) vorsieht, dass Organe und Gewebe nur nach (Hirn-)Todesfeststellung entnommen werden dürfen (TPG 2/§ 3). Hindernis, weil die meist mit dem Sterben 
einer Person einhergehenden Ereignisse und Prozesse der Trauer mit den oft knappen Ressourcen von Intensivstationen kollidieren können, die Bedürfnisse der Angehörigen also mit den Zeitregimen und Notwendigkeiten der Transplantation oft schwer in Einklang zu bringen sind (Kalitzkus 2009; Jensen 2011; Bea 2017). Wie komplex diese Nähe-Distanz-Beziehung zum Themenkomplex Tod und Sterben in der Organspende ist, schlägt sich nicht zuletzt in den immer wieder sichtbaren Versuchen nieder, Organspende in der öffentlichen Wahrnehmung als eine Art ,Feier des Lebens`zu verankern. Mit Annemarie Mol (2002) lassen sich mögliche Kollisionen der Körper- und Todesverständnisse durch deren Multiplizität erklären: Denn die Körper potenzieller Spender:innen sind andere in der Wahrnehmung der Lebenden, in der Logik der Trauer, der Transplantation oder des Marketing. Die Überschreitung der Grenzräume, in denen sie ,andere 'sind, erfordert, wie sich mit Kristeva vermuten lässt, Leistungen der Übersetzung und Koordination, die immer wieder auch neu erprobt werden.

2. Beinhaltet die Organspende eine Zergliederung von menschlichen Körpern in ihre Bestandteile, die tief verankerte Vorstellungen einer Deckungsgleichheit von physischer Materialität und Person in Frage stellen (Lindemann 2003). Zudem kann die Vorstellung über diese Zerlegung mit Gefühlen wie Scham besetzt bzw. mit kulturhistorisch verankerten Konzepten von Strafe verbunden werden (Richardson in Sque und Payne 2007). Der Vorgang selbst wird selten bildlich dargestellt. Eine wichtige Rolle spielt eine Idee von Ästhetik, die das visuell Ansprechende mit dem moralisch Richtigen verbinden (Jensen 2011). So betont eine Informationsbroschüre der BZgA (2016), dass die Organentnahme ,,mit der gleichen chirurgischen Sorgfalt durchgeführt“ würde, ,wie eine normale Operation“ (S. 41). Der „würdige Zustand“ (S. 42) für die Bestattung wird versichert. Zugleich berichten Kritiker:innen über den Zustand von gespendeten Körpern als ,entstellt‘ oder ,ausgenommen', um nicht nur ihrem Unbehagen über die Vorgänge, sondern auch deren moralischer Verwerflichkeit Ausdruck zu verleihen. ${ }^{3}$ Abjekttheoretisch betrachtet wären dies nicht einfach oppositionelle Perspektiven eines Diskurses, sondern stabilisierende kulturelle Annäherungen an diese Möglichkeit des Umgangs mit dem Körper.

3. Betrifft die Spende von Organen die Bestandteile des Körpers selbst, ihre von Blut und anderen Flüssigkeiten durchsetzte und umgebene Umwelt, die uns im Alltag meist verborgen bleibt (Motakef 2011). Die Ästhetik innerer Organe und ihre sinnlich wahrnehmbaren Bestandteile (Temperatur, Haptik oder Geruch) finden nur in bestimmten kulturellen Bereichen, vorwiegend in der medizinisch-technischen Praxis (Solhdju 2015) oder im Genre der Horrorerzählungen (Krüger-Fürhoff 2012) ihren Platz. Getrennt vom Körper beginnen die Organe eine Art Eigenleben (Solhdju 2015), das Organ nach der Entnahme geht als ,die Leber` oder ,das Herz' auf seine Reise durch die Institutionen, nicht aber als Teil einer Person. Mit Blick auf die Angehörigen von Spenderinnen- und Spenderfamilien beschreibt Sharp (2007) die damit verbundenen institutionalisierten Vorgänge als ,Deperso-

\footnotetext{
3 Beschreibungen dieser Art finden sich immer wieder vor allem im Umfeld der Vereinigung KAO (Kritische Aufklärung Organspende, e. V.) und in deren Informationsmaterialien und Internetauftritten (vgl. z. B. KAO о.J.).
} 
nalisierung', die einerseits für ein funktionierendes Transplantationssystem notwendig seien, andererseits Individuen auf „sources of reusable parts“ (S. 58) reduziere. Eben diese Transformation sei für viele transplantierte Patient:innen zugleich notwendig, da sich für deren personelles Selbstverständnis die Narration des ,Weiterlebens ' im Anderen oft nur bedingt eigne (S. 58). Mit der Theorie des Abjekten wären eben jene klaren Abgrenzungen der Sphären der Spender:innen von jenen der Empfänger:innen auch als kulturelle Einebnung jenes prekären ,Dazwischenseins ' transplantierter beziehungsweise zu transplantierender Organe beschreibbar.

4. Birgt die Transplantation eine Auflösung von Grenzen (wie Leben/Tod und Mein Körper/Dein Körper), die wir in unseren kulturellen Vorstellungsräumen gerne (binär) setzen (Giesen 2010, S. 9f.). Diese werden sowohl durch das Konzept des Hirntods als auch durch die Übertragung von Körperteilen aufgewühlt und durcheinandergebracht (Lindemann 2003; Esser und Kersting 2017). Der Spender:innenkörper befindet sich in einem Zustand, der sich mit Giesen (2010) als „Zwischenlage“ beschreiben lässt; er ähnelt den Lebenden ebenso wie den Toten und hat in seiner empirischen Uneindeutigkeit konstitutive Bedeutung (S. 29). Dieses konstituierende Element finden wir im Changieren der Organe zwischen Heldennarrativen und lebensrettendem Material (Sharp 2007) ebenso wie in immer neuen Verordnungen und Aushandlungen um den Hirntod als Tod des Menschen (Deutscher Ethikrat 2015). Kritiker:innen stellen auf eben jene Uneindeutigkeit ab, wenn sie vom „Tod bei lebendigem Leib“ und „Tötung“ ihrer Angehörigen durch die Entnahme sprechen (KAO o.J.).

Zusammengefasst wären jene Bilder von Fahrrädern, Frühlingsgärten und Ausweisen, die in den Kampagnen für Organspende stehen, in dieser Lesart Elemente von Aushandlungs- und Einhegungsprozessen einer Praxis, die an wesentliche, anthropologische Grundelemente des menschlichen Daseins und Zusammenlebens rührt: darunter die Unversehrtheit des Körpers, die Einheit von Körper und Person, die Einheit des Körpers und seiner Teile sowie die Frage nach den Grenzziehungen zwischen Leben und Tod. Artefakte wie der Organspendeausweis sind damit auch Ergebnis eines konstitutiven Ordnungsprozesses im Umgang mit dem Ungefähren (Giesen 2010).

\section{Diskussion und Konklusion: Kulturen der Organspende als Kulturen des Todes}

Was können wir von Kristevas Konzept des Abjekten über Organspende lernen? Mit Kristeva gelingt es, den Blick auf die kulturellen Sicherheitsmaßnahmen zu lenken, die mögliche Bedrohungen der Grenze von Subjekt und Ordnung in einen kulturellen Rahmen bannen und damit auch stabilisierende und konstituierende Momente aufweisen. So sind es eben jene ambivalenten Bereiche, das fortwährende Spiel der Annäherung und Distanzierung und ihre Übersetzungen in Bilder, Narrationen und Objekte (wie den Organspendeausweis), die die Kulturen der Organspende konstitutiv (mit-)begründen. Dabei ist es vor allem der Spender:innenkörper, 
mit seiner Sterblichkeit und expliziten Materialität, dem wir uns mit größter Vorsicht annähern, wenn Organspende als gesellschaftliche Praxis debattiert wird. Dies inkludiert die Bedeutung der Ästhetik, die Jensen (2011) in der Pflege hirntoter Spender:innen findet, spiegelt sich in Bildern und Metaphern von Fahrrädern, Pflanzen oder Schmetterlingen (s. oben) oder im häufig thematisierten schwarzen Humor von medizinischem Personal (Bleakley 2017). Auch der in den deutschen Kampagnen so prominent platzierte Ausweis kann als Requisit der Einhegung abjekter Konfrontationsräume gedeutet werden, da er es ermöglicht, die Vorgänge am Spender:innenkörper in (vermeintlich) einfache, bekannte Handlungen, wie das Unterschreiben eines Dokuments, zu transformieren (Böhrer 2018). Die kleine Karte erlaubt Distanz und Nähe zugleich, sie ermöglicht performative Strategien, verbindet als Grenzobjekt (Leigh-Star und Griesemer 1989) das Unvorstellbare mit der Welt der täglichen Routine. Zugleich werden jene abjekten Elemente auf das sachlich nötigste reduziert, das Abjekte auf eine kleine, tragbare Fläche gebannt. Dies ist insbesondere mit Blick darauf interessant, dass das Konstrukt ,Ausweis“ in der Umsetzung der Transplantation eine nur untergeordnete Rolle spielt, ${ }^{4}$ zugleich aber intensiv beworben wird.

Mit Larissa Pfaller (2020) können wir sehen, dass das Konzept des Abjekten, obgleich es in der Soziologie bisher nur am Rande Beachtung gefunden hat, hilfreich sein kann, um tiefere kulturelle Bedeutungen und Symboliken sozialer Praktiken zu erforschen und ein besseres Verständnis für ihre psychosoziale Dynamik zu generieren. Der Schlüssel für die produktive Begegnung mit dem Abjekten sei in Strategien der Anerkennung und des Empowerments zu suchen (Pfaller 2020, S. 827). Übertragen auf unseren hier vorliegenden Fall könnte es sich also als hilfreich erweisen, Ambivalenzen nicht in erster Linie durch Aufklärung und Überzeugungsarbeit überwinden zu wollen, sondern als integralen Bestandteil der Praxis der Transplantation selbst anzuerkennen und jenen Bereichen, die sich durch ihre Abwesenheit in der affirmativen Kommunikation und Anwesenheit in Gegennarrationen auszeichnen, mit größerer Offenheit zu begegnen. Dies würde auch beinhalten anzuerkennen, dass eine solche Verbindung toter und lebender Körper, wie sie mit der Organspende geschaffen wurde, nicht nur ein Fest des Lebens, sondern Bestandteil von Trauerkulturen und Ausdruck der Anerkennung der Vergänglichkeit, Verletzlichkeit und Materialität unserer Körperlichkeit ist. Dies erfordert aber auch eine entsprechende Anerkennung der Spender:innen und aller Ambivalenzen, die dieser Umgang mit ihren Körpern mit sich bringt.

Funding Open Access funding enabled and organized by Projekt DEAL.

Open Access Dieser Artikel wird unter der Creative Commons Namensnennung 4.0 International Lizenz veröffentlicht, welche die Nutzung, Vervielfältigung, Bearbeitung, Verbreitung und Wiedergabe in jeglichem Medium und Format erlaubt, sofern Sie den/die ursprünglichen Autor(en) und die Quelle ordnungsgemäß nennen, einen Link zur Creative Commons Lizenz beifügen und angeben, ob Änderungen vorgenommen wurden.

\footnotetext{
${ }^{4}$ Dies zeigt sich bspw. in den jährlichen Statistiken zur Organspende, die von der Deutschen Stiftung Organtransplantation herausgegeben werden: https://dso.de/organspende/statistiken-berichte/organspende.
} 
Die in diesem Artikel enthaltenen Bilder und sonstiges Drittmaterial unterliegen ebenfalls der genannten Creative Commons Lizenz, sofern sich aus der Abbildungslegende nichts anderes ergibt. Sofern das betreffende Material nicht unter der genannten Creative Commons Lizenz steht und die betreffende Handlung nicht nach gesetzlichen Vorschriften erlaubt ist, ist für die oben aufgeführten Weiterverwendungen des Materials die Einwilligung des jeweiligen Rechteinhabers einzuholen.

Weitere Details zur Lizenz entnehmen Sie bitte der Lizenzinformation auf http://creativecommons.org/ licenses/by/4.0/deed.de.

\section{Literatur}

Amelang, Katrin. 2014. Transplantierte Alltage. Zur Produktion von Normalität nach einer Organtransplantation. Bielefeld: Transcript.

Arp, Doris. 2011. Organspende. Deutschlandfunk Kultur. https://www.deutschlandfunk.de/organspendepflicht-oder-geschenk.724.de.html?dram:article_id=100236. Zugegriffen: 25. Mai 2021.

Bea, Sara. 2017. No heroics, please. https://era.ed.ac.uk/handle/1842/23376. Zugegriffen: 27. Mai 2021.

Bleakley, Alan. 2017. Thinking with metaphors in medicine: the state of the art. London/New York: Routledge.

Böhrer, Annerose. 2018. Entscheiden, Ankreuzen, Verfügen. In Herumschnüffeln - aufspüren - einfühlen, Hrsg. Ronald Hitzler, et al., 355-368. Essen: Oldib.

Bundesministerium der Justiz und für Verbraucherschutz/Bundesamt für Justiz. 2007. Transplantationsgesetz in der Fassung der Bekanntmachung vom 4. September 2007 (BGB1. I S. 2206). https://www. gesetze-im-internet.de/tpg/. Zugegriffen: 8. März 2020.

BZgA. 2016. Informieren, entscheiden, ausfüllen. https://www.bzga.de/presse/pressemitteilungen/201610-27-informieren-entscheiden-ausfuellenneue-informationskampagne-zum-thema-organ-undgew/. Zugegriffen: 25. Mai 2021.

BZgA. Ohne Angaben. Plakatserie „Organspende, die Entscheidung zählt“. https://www.organspendeinfo.de/mediathek/plakatserie.html. Zugegriffen: 25. Mai 2021.

Danek, Sabine. 2010. Pro Organspende. https://page-online.de/kreation/pro_organspende_du_bekommst_ alles_von_mir/. Zugegriffen: 25. Mai 2021.

Deutscher Ethikrat. 2015. Hirntod und Entscheidung zur Organspende. Hamburg: Bartos Kersten Printmediendesign.

Donate life. Ohne Angaben. National donate life month. https:/www.donatelife.net/ndlm/. Zugegriffen: 25. Mai 2021.

DSO. 2020. Mögliche Organspender. Statistiken zur Organspende. https://dso.de/DSO-Infografiken/M \%C3\%B6gliche\%20OS.png. Zugegriffen: 17. Aug. 2021.

Esser, Andrea, und Daniel Kersting. 2017. Der Tod ist das Ende dieses Lebens. In Transmortalität, Hrsg. Antje Kahl, et al., 196-228. Weinheim \& Basel: Beltz Juventa.

Giesen, Bernhard. 2010. Zwischenlagen. Weilerwist: Velbrück Wissenschaft.

Hansen, Solveig, und Silke Schicktanz. 2018. Bilder fürs Leben. In Die Krise der Organspende, Hrsg. Andrea Esser, et al., 85-114. Berlin: Duncker \& Humblot.

Hansen, Solveig, Larissa Pfaller, und Silke Schicktanz. 2020. Critical analysis of communication strategies in public health promotion: an empirical-ethical study on organ donation in Germany. Bioethics https://doi.org/10.1111/bioe.12774.

Jensen, Anja. 2011. Orchestrating an Exceptional Death. København: Institut for Antropologi. Ph.D. Series, No. 69. https://publichealth.ku.dk/staff/?pure=en\%2Fpublications\%2Forchestrating-anexceptional-death(a3e1ff6f-232c-44cc-a650-0424eb475713).html. Zugegriffen: 18. Aug. 2021.

Kalitzkus, Vera. 2009. Dein Tod, mein Leben: Warum wir Organspenden richtig finden und trotzdem davor zurückschrecken. Frankfurt a. M.: Suhrkamp.

KAO. Ohne Angaben. Organspende: Die verschwiegene Seite. Kritische Aufklärung über Organspende e. V. https://initiative-kao.de/. Zugegriffen: 27. Mai 2021.

Kristeva, Julia. 1982. The powers of horror: an essay on abjection. New York: Columbia University Press. Krüger-Fürhoff, Irmela. 2012. Verpflanzungsgebiete: Wissenskulturen und Poetik der Transplantation. München: Willhelm Fink.

Lakoff, George, und Mark Johnson. 2003. Metaphors we live by. Chicago, London: University of Chicago Press. 
Leigh-Star, Susan, und James Griesemer. 1989. Institutional ecology, 'translations' and boundary objects: amateurs and professionals in Berkeley's Museum of Vertebrate Zoology, 1907-39. Social Studies of Science 19(4):387-420. https://doi.org/10.1177/030631289019003001.

Lindemann, Gesa. 2003. Beunruhigende Sicherheiten: Zur Gense des Hirntodkonzepts. Konstanz: UVK.

Mol, Annemarie. 2002. The body multiple: ontology in medical practice. Durham: Duke University Press. Motakef, Mona. 2011. KörperGabe: Ambivalente Ökonomien der Organspende. Bielefeld: transcript.

New Hannover Regional Medical Center. Ohne Angaben. Art for organ donation. https://web.archive. org/web/20200810082924/https://www.nhrmc.org/patients/care-decisions/organ-donation/bike-art. Zugegriffen: 10. Aug. 2020.

Pfaller, Larissa. 2020. Theorizing the virus: abjection and the COVID-19 pandemic. International Journal of Sociology and Social Policy 40(9/10):821-829. https://doi.org/10.1108/IJSSP-06-2020-0243.

Richardson, Ruth. 2007. Human dissection and organ transplantation in historical context. In Organ and tissue donation, Hrsg. M. Sque, S. Payne. Maidenhead/New York: Open University Press.

Schaper, Manuel, Solveig Hansen, und Silke Schicktanz. 2018. Überreden für die gute Sache? Techniken öffentlicher Gesundheitskommunikation und ihre ethischen Implikationen. Ethik in der Medizin 31:23-44. https://doi.org/10.1007/s00481-018-0507-7.

Sharp, Lesley. 2007. Bodies, commodities and biotechnologies: death, mourning, and scientific desire in the realm of human organ transfer. New York: Columbia University Press.

Solhdju, Katrin. 2015. Konzepte des Lebendigen und Kulturen des Interesses. In Das Leben vom Tode her, Hrsg. Katrin Solhdju, Ulrike Vedder, 163-181. Paderborn: Wilhelm Fink. https://doi.org/10.30965/ 9783846757468_012.

Woodward, Sophie. 2019. Material methods. L.A./London/New Delhi/Singapore/Washington DC/ Melbourne: SAGE. 